\title{
Médiévales
}

Langues, Textes, Histoire

72 | printemps 2017

Roman du Genji et société aristocratique au Japon

\section{Entre Orient et Occident, les légendes médiévales d'Alexandre le Grand dans l'historiographie récente}

Anna Caiozzo

\section{(2) OpenEdition}

Journals

Édition électronique

URL : https://journals.openedition.org/medievales/8131

DOI : 10.4000/medievales.8131

ISSN : $1777-5892$

Éditeur

Presses universitaires de Vincennes

Édition imprimée

Date de publication : 22 juin 2017

Pagination : 175-194

ISBN : 978-2-84292-612-0

ISSN : 0751-2708

Référence électronique

Anna Caiozzo, « Entre Orient et Occident, les légendes médiévales d'Alexandre le Grand dans

I'historiographie récente », Médiévales [En ligne], 72 | printemps 2017, mis en ligne le 28 février 2019,

consulté le 22 avril 2022. URL : http://journals.openedition.org/medievales/8131 ; DOI : https://

doi.org/10.4000/medievales.8131 


\section{Entre Orient et Occident, les légendes médiévales d'Alexandre le Grand dans I'historiographie récente}

\section{Alexandre, une figure transculturelle}

La figure d'Alexandre le Grand a été promue au rang de mythe par trois types de récits : par ceux (perdus pour l'essentiel) de ses compagnons (Eumène, Callisthène, Charès de Mytilène, Néarque, etc.), par ceux des historiens postérieurs (Arrien, Quinte-Curce, Plutarque, Diodore de Sicile), et par la version légendaire enfin, celle du III ${ }^{\mathrm{e}}$ siècle en grec du PseudoCallisthène et de son Roman d'Alexandre, qui fut à la source des versions établies entre le $\mathrm{IV}^{\mathrm{e}}$ et le $\mathrm{VI}^{\mathrm{e}}$ siècle, d'abord en arménien, en géorgien, en pahlavi, puis en syriaque. La version syriaque inspira celles en arabe, en éthiopien et en hébreu. Ainsi se diffusa, du monde byzantin à l'Europe occidentale, du Levant à l'Éthiopie (et à l'Afrique ${ }^{1}$ ), et de la Perse au monde arabe, la légende d'un jeune conquérant du monde, ivre d'ambition, en quête de gloire et de richesses, vainqueur du roi de Perse et des souverains de l'Inde, et dont la geste spectaculaire embrassait l'œkoumène de l'océan des ténèbres à la muraille de Gog et Magog, tout en révélant successivement les merveilles de la Création. Par la suite, le chant des bardes orientaux diffusa la légende en Asie centrale, en Mongolie, en Inde où perdure longtemps le souvenir de l'une des figures les plus fascinantes que le monde ait porté, mais entièrement construite par l'imaginaire des hommes.

L'attractivité d'une telle figure est liée en grande partie à son ambiguïté : personnage ambitieux et séduisant, prototype du héros, tragiquement fauché à trente ans, conquérant du monde, Alexandre fit en Orient comme en Occident rêver des générations de rois et leurs aristocratiques sujets. Alexandre est en effet un personnage polymorphe, bénéfique et

1. Le Roman d'Alexandre à Tombouctou. Histoire du Bicornu. Le manuscrit interrompu, éd. G. Bohas, A. SAguer et A. Sinno, Paris, 2012. 
maléfique, adulé et détesté, guerrier et conquérant, navigateur ${ }^{2}$, héros courtois, maître de magie et initié du Sirr al-asrār ${ }^{3}$, prophète dans certaines traditions. Si sa geste est à la fois ancrée dans le réel et dans le mythe, on est en droit de s'interroger sur la pérennité d'un tel succès durant des siècles ; cette fascination du $\mathrm{X}^{\mathrm{e}}$ au $\mathrm{XVI}^{\mathrm{e}}$ siècle, tant de la part des écrivains - orientaux comme occidentaux - que de leur public, ne réside-t-elle pas plus dans la malléabilité d'une figure dualiste, marquée d'un côté par l'hybris, mais portant de l'autre le rêve qui est bien celui de tous les hommes : boire à la fontaine de vie pour vaincre la mort, cette insigne marque de notre humanité ${ }^{4}$ ?

L'essor des études médiévales aidant, chacun voulant étudier son propre «Alexandre», ce personnage est devenu depuis une vingtaine d'années le thème en vogue de nombreux travaux personnels ou collectifs, croisant traditions orientales et occidentales.

Certes, de nombreux travaux avaient déjà approché le personnage : ceux d'Emilio García Gómez sur le roman arabe ${ }^{5}$, de Wallis Budge sur le texte éthiopien ${ }^{6}$, d'Israël Levi sur Alexandre dans le Talmud ${ }^{7}$, ou encore d'Albert Wolohojian sur la tradition arménienne ${ }^{8}$. Une des études inoubliables demeure celle de George Gary qui, en 1953, grâce à David J. A. Ross, nous laissait son Medieval Alexander publié en $1956^{9}$. Alexander Runni Anderson abordait celle des fameux Gog et Magog ${ }^{10}$, alors que David White explicitait le problème des races monstrueuses ${ }^{11}$. Le voyage est, en

2. M.-A. PiEMONTESE, «Alexandre le circumnavigateur dans le roman persan de Tartusi », dans F. DE POLIGNAC éd., Alexandre le Grand, figure de l'incomplétude. Mélanges de l'École Française d'Athènes et de Rome, 112/1 (2001), p. 97-112.

3. Voir infra, n. 19.

4. P. FrANKE, « Drinking from the Water of Life - Nizāmī, Khizr and the Symbolism of Poetical Inspiration in Later Persianate Literature », dans J.-C. BÜRGEL et C. VAN RUYMBEKE éd., A Key to the Treasure of the Hakìm: Artistic and Humanistic Aspects of Nizāmī Ganjavī's Khamsa, Leyde, 2011, p. 107-126 ; M. CASARI, « Nizāmī’s Cosmographic Vision and Alexander in Search of the Fountain of Life », ibid., p. 95-106.

5. E. GARCÍA GómeZ, Un texto arabe occidental de la leyenda de Alejandro, Madrid, 1929.

6. E. A. W. Budge, The History of Alexander The Great Being the Syriac Version of The Pseudo-Callisthenes, Cambridge, 1889, et aujourd'hui P. KOTAR, Der syrische Alexanderroman, Hambourg, 2013.

7. I. LEVI, «La légende d'Alexandre dans le Talmud», Revue des études juives, 2 (1881), p. 293-300 ; ID., « La légende d'Alexandre dans le Talmud et le Midrash », Revue des études juives, 7 (1883), p. 78-93.

8. A. H. WolohoJin, The Romance of Alexander the Great by Pseudo-Callisthenes (from the Armenian), New York, 1969.

9. G. CARY, The Medieval Alexander, éd. D. J. A. Ross, Cambridge, 1956, rééd. New York, 1987.

10. A. RunNi ANDERSon, Alexander's Gate, Gog and Magog, and the Inclosed Nations, Cambridge, 1932.

11. D. G. White, Myth of the Dog Men, Chicago, 1991. 
effet, d'abord marqué par le merveilleux : plantes, races tératomorphes, qui s'ajoutent aux composantes de l'imaginaire occidental comme oriental.

John Boyle, lui, dès 1977, montrait la nécessité de confronter les traditions en Orient et en Occident ${ }^{12}$. C'est ainsi qu'en 1997 JohannChristoph Bürgel proposait une réflexion sur les conceptions du pouvoir ${ }^{13}$, suivi, en 1999, par le colloque organisé par Claire Kappler, François Suard et Laurence Harf-Lancner à l'Université Paris 10 (Ouest-Nanterre), Alexandre le Grand dans les littératures occidentales et proche-orientales ${ }^{14}$. La rencontre posait les bases des problèmes soulevés par la tradition littéraire, le rôle des milieux syriaques, l'ambiguïté d'une figure héroïque reconstruite, à la fois exaltée pour sa geste dans les traditions médiévales tant orientales qu'occidentales, mais aussi décriée comme une figure de l'ambition démesurée, incarnant l'hybris et la finitude de l'homme quelles que soient la puissance et l'ambition qui le caractérisent. Les années 20002010 sont d'ailleurs riches de réflexions croisées ${ }^{15}$ et les travaux sur la légende orientale d'Alexandre se multiplient.

Alexandre est abordé comme héros transnational et transculturel, depuis l'Himalaya - où sa légende est restée vivace et inspira à Rudyard Kipling «L'homme qui voulut être roi ${ }^{16}{ }^{»}-$ jusqu'à l'Asie centrale. Il fait en somme partie du patrimoine de l'humanité, comme l'ont montré Roberte Hamayon et Françoise Aubin ${ }^{17}$.

\section{Alexandre d'Orient}

En Orient où naquit sa légende, Alexandre le Grand est une figure complexe, à la fois historique comme conquérant et destructeur de l'Empire perse, mais aussi imaginaire aux accents religieux, intégré aux traditions

12. J. A. BoyLE, «The Alexander Romance in the East and West », Bulletin of the John Rylands University Library of Manchester, 60 (1977), p. 19-20 ; W. J. AERTS et al., Alexander the Great in the Middle Ages, Nimègue, 1978.

13. J.-C. BÜRGEL éd., The Problematics of Power, Eastern and Western Representations of Alexander the Great, Berne-Berlin, 1997.

14. L. HARF-LANCNER, C. KAPPLER et F. SuARD éd., Alexandre le Grand dans les littératures occidentales et proche-orientales, Actes du colloque de Paris, 27-29 novembre 1997, Nanterre, 1999.

15. F. DE POLIGNAC éd., Alexandre le Grand, figure de l'incomplétude...

16. A. AKASOY, «Alexander in the Himalayas Competing Imperial Legacies in Medieval Islamic History and Literature », Journal of the Warburg and Courtauld Institutes, 72 (2009), p. 1-20. Voir aussi N. Gopala PILlaI, "Skanda: The Alexander Romance in India", Proceedings of the All-India Oriental Conference, 9 (1937), p. 955 -976.

17. F. Aubin et R. HAMAYON, Alexandre, César et Gengis Khan dans les steppes d'Asie centrale, Paris, 2001, p. 73-106. 
coraniques ${ }^{18}$ en tant que prophète que certains voudraient reconnaître dans le fameux Dhü'l Qarnayn de la sourate XVIII/60-102 ${ }^{19}$.

Si le terrain avait été défriché par Mario Grinaschi ${ }^{20}$, c'est cette figure «arabe» qu'explore Faustina Aerts dans Alexander Magnus Arabicus. The Alexander Tradition through Seven Centuries : from PseudoCallisthenes to Șūr $\bar{l}^{21}$. L'ouvrage est une somme désormais incontournable pour comprendre la naissance d'une tradition littéraire et ses différents aspects dans les pas de celle du Pseudo-Callisthène et de ses traductions du syriaque ou du byzantin à l'arabe. Toutes les versions et traditions en arabe sont passées en revue de façon exhaustive et systématique : d'une part, les chroniques ou ouvrages historiques, mais aussi les différentes versions de la romance dont celle qui survécut en al-Andalus (chapitre 1); d'autre part, les ouvrages appartenant à la tradition littéraire des miroirs des princes (chapitre 2). Enfin, les deux traditions importantes, celle du fameux Dhū'l Qarnayn (chapitre 3) et de la Sīra al-Iskandar (chapitre 4), où sont décrits les différents aspects de la légende populaire évoquant tant les personnages associés au mythe que les lieux emblématiques liés à sa geste; la seconde partie, précisément consacrée à la Sīra d'un point de vue littéraire, évoque d'autres caractéristiques thématiques.

Dans le monde iranien, la légende d'Alexandre se complexifie: héritière d'une historiographie négative, celle du destructeur de l'empire sassanide $^{22}$, tout en en faisant aussi un conquérant apparenté aux rois de

18. Voir n. 37.

19. N. O Brown, «The Apocalypse of Islam», Social Text, 8 (1983-1984), p. 155171 ; B. WHEELER, « Moses or Alexander ? Early Islamic Exegesis of Qur'an 18 :60-65 », Journal of Near Eastern Studies, 57 (1998), p. 191-215 ; et, plus récemment sur cette sourate, M. Chasseur, « Oriental Elements in Surat al Kahf», Annali di Scienze Religiose, 1 (2008), p. 255-289.

20. Mario Grinaschi a imprimé sa marque à la perception d'Alexandre arabe et sur la littérature hermétique : voir surtout «Les "Rasā'il 'Āristātālīsa ilā-l-Iskandar" de Sālim Abū-l- 'Alā' et l'activité culturelle à l'époque omayyade », Bulletin d'études orientales, 19 (1965-1966), p. 7-83 ; ID., «Le roman épistolaire classique conservé dans la version arabe de Salim Abu-1-“Ala »”, Le Muséon, 80 (1967), p. 211-264 ; ID., « L'origine et les métamorphoses du Sirr al-asrar », Archives d'Histoire doctrinale et littéraire du Moyen Âge, 43 (1976), p. 7-112 ; et enfin ID., «La figure d'Alexandre chez les Arabes et sa genèse », Arabic Science and Philosophy, 3 (1993), p. 205-234.

21. F. DOUfIKAR-AERTS, Alexander Magnus Arabicus. The Alexander Tradition through Seven Centuries : from Pseudo-Callisthenes to Sūrū, Paris/Louvain, 2010.

22. J. DARMESTETER, « La légende d'Alexandre chez les Parses », dans Essais orientaux, Paris, 1883, p. 227-250 ; ID., «Alexandre le Grand dans le Zand-Avesta », Lectures de l'Académie des Inscriptions et Belles-Lettres, 11 (1892), p. 190-196. Voir aussi l'excursus de M. Southgate, «Alexander in Pahlavi Literature», dans Iskandarnamah : A Persian Medieval Alexander-Romance, New York, 1978, p. 186-189. 
Perse, et donc dépositaire de la gloire royale ${ }^{23}$, le personnage se double d'un initié, voire d'un maître de magie ${ }^{24}$.

Ainsi, la poésie épique en persan, s'inspirant à la fois du PseudoCallisthène, des faits historiques et de l'apport de l'hermétisme arabe, en fit un personnage héroïque exceptionnel et, comme l'a montré Charles-Henri de Fouchécour ${ }^{25}$, un modèle pour les rédacteurs de miroirs des princes jusqu'au $X^{\mathrm{e}}$ siècle !

Les deux auteurs qui ont révélé la figure d'Alexandre sont deux poètes : Firdawsī de Ṭūs, d'une part, au début $\mathrm{du} \mathrm{XI}^{\mathrm{e}}$ siècle dans son Histoire des rois de Perse ou Shāh nāma ${ }^{26}$; et d'autre part, au début XII ${ }^{\mathrm{e}}$ siècle $^{27}$, Nizāāmī de Ganja ${ }^{28}$, auteur d'un Iskandar nāma, suivis de l'Indien Khusraw Dihlavī à la fin du XIII ${ }^{\mathrm{e}}$ et au début du XIV ${ }^{\mathrm{e}}$ siècle ${ }^{29}$. La célébration du millénaire de l'œuvre de Firdawsī jointe à l'intérêt suscité récemment par celle de Nizāāī de Ganja dans les traces des travaux comparatistes lancés par J.-C. Bürgel ${ }^{30}$, ont permis de continuer d'en explorer la figure et de relire les vieux mythes de façon rafraîchissante. La localisation de la fameuse muraille des Gog et

23. G. GNOLI, « Note sullo "xvaranah" », dans Hommages et Opera Minor, Orientalia J. Duchesne-Guillemin, Emerito Oblata, Acta Iranica, 9, Leyde, 1984, p. 207-218.

24. Sur le rapport aux sciences occultes, J.-C. BÜRGEL, "Occult Sciences in the Iskandarnameh of Nizâmî », dans K. TALATTOF et J. W. CLINTON éd., The Poetry of Nizâmî Ganjavi, Knowledge, Love, and Rhetoric, New York, 2000, p. 129-139.

25. C.-H. DE FOUCHÉCOUR, Le Sage et le prince en Iran médiéval. Morale et politique dans les textes littéraires persans. $X^{e}$-XIII ${ }^{e}$ siècles, Paris, 2009.

26. Sur le Shāh nāmah et les nombreux travaux qui en assurent la promotion depuis 2004 à Cambridge autour de Charles Melville, on peut voir la bibliographie de la base de données http://shahnama.caret.cam.ac.uk/new/jnama/bibliography.

27. Voir les travaux de C. KAPPLER, «Alexandre et les merveilles dans le Livre des rois de Firdawsî », dans Et c'est la fin pour quoy nous sommes ensemble. Hommage à Jean Dufournet, Littérature, Histoire et Langue du Moyen Âge, Paris, 1993, t. 2, p. $759-773$; EAD., "Alexandre dans le Shāh Nāmeh de Firdawsî: de la conquête du monde à la découverte de soi », dans BÜRGEL éd., The Problematics..., p. 165-190 ; Y. YAMANAKA, «Ambiguïté de l'image d'Alexandre chez Firdawsī : les traces des traditions sassanides dans Le Livre des Rois », dans L. HARF-LANCNER, C. KAPPLER et F. SUARD éd., Alexandre le Grand..., p. 341353.

28. J.-C. BÜRgel, Nizami. Das Alexanderbuch, Munich, 1991 ; C. SaCCOnE, Il libro della fortuna d'Alessandro, Eqbâlnâmeh, Milan, 1997 ; W. C. Clarke, The Sikandar Nâma e Barâ, Londres, 1881.

29. M. PiemonteSe, Gli "Otto Paradisi" di Amir Khusrau da Delhi, una lezione persiana del "Libro di Sindbad" fonte del "Peregrinaggio" di Cristoforo Armeno, Rome, 1995, p. 317-418.

30. J.-C. Bürgel et C. VAn RuymbeKe éd., A Key... 
Magog $^{31}$, dont l'illustration préoccupe enfin les chercheurs ${ }^{32}$, est identifiée en 2010 par Emeri van Donzel et Andrea Schmidt ${ }^{33}$. La quête du paradis et de la source de vie ${ }^{34}$ complètent le beau portrait d'al-Khādir par Françoise Aubaile-Sallenave ${ }^{35}$. En outre, les traductions récentes de l'Iskandar nāmā anonyme par Minoo Southgate ${ }^{36}$ et du Dārāb nāma de Țarsūsī par Marina Gaillard ${ }^{37}$ donnent des clefs supplémentaires pour aborder le héros.

La dimension comparatiste perdure et, en 2011, David Zuwiyya, dont les précieux travaux recensent les légendes islamiques ${ }^{38}$, convoquait les spécialistes des textes latins et des traditions juives, syriaques, arabes, perses, coptes, éthiopiennes et occidentales (France, Espagne, GrandeBretagne, Allemagne, Italie, Scandinavie), pour évaluer de nouvelles perspectives sur la figure héroïque ${ }^{39}$. En 2012, Richard Stoneman ${ }^{40}$, Kyle Erickson et Ian Netton présentent un nouveau volume comparatiste, The Alexander Romance in Persia and the East ${ }^{41}$, où, en dehors de l'apport notable de D. Zuwiyya, F. Aerts, M. Gad et E. Cottrell sur les sources et les variantes de la légende dans les textes arabes, on peut signaler quelques articles originaux montrant l'inscription de la légende dans l'espace

31. A. RunNI ANDERSON, «Alexander and the Caspian Gates », dans Transactions and Proceedings of the American Philological Association, 59 (1928), p. 130-163 ; ID., Alexander's Gate, Gog and Magog and the Inclosed Nations, Cambridge, 1932 ; P. CUNEO, «Le mura di Darbent, Note sulla topografia e la morfoligia urana di una città-stato del limes islamico nell'area caucasica », Rivista degli Studi orientali, 59 (1985), p. 57-75 ; et plus récemment C. GENEQUAND, «Autour de la ville de Bronze : d'Alexandre à Salomon », Arabica, 39/3 (1992), p. 328-345.

32. G. VAN DEN BERG, «Descriptions and Images - Remarks on Gog and Magog in Nizāmī’s Iskandar Nāma, Firdawsī’s Shāh Nāma and Amīr Khusraw’s A'̄̄na-yi Iskandarī », dans J.-C. BÜRGEL et C. VAN RUYMBEKE éd., A Key..., p. 77-94.

33. E. VAN DONZEL et A. SCHMIDT, Gog and Magog in Early Eastern Christian and Islamic Sources, Sallam's Quest for Alexander's Wall, Leyde, 2010.

34. P. FrAnKE, « Drinking... »; M. CASARI, « Nizāmī’s Cosmographic Vision... »; et surtout C. SACCONE, Un profeta e santo-iniziatore, Elia al Khidr nella tradizione musulmana, Milan, 2004.

35. F. Aubaile-SAllenAVE, «L'homme au manteau vert en pays musulman: ses fonctions, ses caractères, sa diffusion », Res orientales, 14 (2002), p. 11-35.

36. M.Southgate, Iskandarnamah : A Persian Medieval Alexander-Romance, New York, 1978 ; EAD., « Portrait of Alexander in Persian Alexander-Romances in the Islamic Era », Journal of the Oriental American Society, 97/3 (1977), p. 278-284.

37. M. GAILlard, Le Dârab Nâmeh d'Abû Tâher Tartusi, Paris, 2005.

38. Z. D.ZUWIYYA, Islamic Legends concerning Alexander the Great, Binghamton, 2001.

39. Z. D. ZuWIYYA éd., A Companion to Alexander Literature in the Middle Ages, Leyde, 2011.

40. R. Stoneman, Alexander the Great : A Life in Legend, New Haven, 2008.

41. R. Stoneman, K. ERICKson et I. Netton éd., The Alexander Romance in Persia and the East, Groningue, 2012. 
turc $^{42}$, dans l'espace indien ${ }^{43}$, les connexions avec la Chine ${ }^{44}$, les traits de la légende dans la littérature juive ou rabbinique ${ }^{45}$, dans la littérature copte $^{46}$, et surtout l'impact de la légende dans l'art de l'Asie centrale ${ }^{47}$, ou des éléments de la légende dans l'art ${ }^{48}$. On doit surtout noter que les auteurs ne citent pas un certain nombre de travaux les ayant précédés ou qui recoupent de façon criante leur propos ; les travaux des universitaires français, notamment, sont absents, un fait inquiétant mais de plus en plus répandu dans la littérature anglo-saxonne.

\section{Alexandre d'Occident}

À la vitalité certaine des travaux des orientalistes, on peut désormais opposer ceux des projets «occidentalistes", Medieval Alexander à l'Université de Rochester ${ }^{49}$, et surtout les fruits d'une ANR en une collection de huit volumes intitulée Alexander Redivivus chez Brepols (2011-2014), dirigée par Catherine Gaullier-Bougassas, Margaret Bridges, Corinne Jouanno et Jean-Yves Tilliette, qui retrace la tradition médiévale d'Alexandre sous tous ses aspects, sans exclure d'ailleurs quelques aspects orientalistes lorsque le thème s'y prête.

Des quatre premiers volumes qui forment un tout, l'un est dédié à l'introduction de la figure d'Alexandre et de la matière d'Alexandre dans les œuvres des auteurs du Moyen Âge, un aspect illustré par un deuxième volume d'édition de textes des $\mathrm{XIII}^{\mathrm{e}}$ et $\mathrm{XIV}^{\mathrm{e}}$ siècles où dominent les

42. C. Jounnno, «The Persians in Late Byzantine Alexander Romances: Portrayal under Turkish Influences », dans R. STONEMAN, K. ERICKSON et I. NETTON éd., The Alexander Romance..., p. 105-116 ; H. Boeschoten, « Adventures of Alexander in Medieval Turkish », ibid., p. 117-126.

43. W. BALL, «Some Talk of Alexander Myths and Politics in the North-West Frontier of British India », dans R. StONEMAn, K. ERICKSON et I. NETTON éd., The Alexander Romance..., p. 127-160 ; A. Szalc, «In Search of the Water of Life : The Alexander Romance and Indian Mythology », ibid., p. 327-338.

44. Y. YAMANAKA, «The Islamized Alexander in Chineses Geographies and Encyclopaedias », dans R. StOnEMAn, K. ERICKSON et I. NETTON éd., The Alexander Romance..., p. 263-276.

45. A. Kleczar, "The Kingship of Alexader the Great in the Jewish Versions of Alexander Narratives », dans R. STONEMAn, K. ERICKSON et I. NETTON éd., The Alexander Romance...,p. 339-348 ; O. AMITAY, «Alexander in Bavli Tamid : In Search for a Meaning », ibid., p. 349-368.

46. L. S. B. MacCoull, « Aspects of Alexander in Coptic Egypt », dans R. STONEMAN, K. ERICKSON et I. NETTON éd., The Alexander Romance..., p. 255-262.

47. O. PALAGIA, «The Impact of Alexander the Great in the Art of Central Asia », dans R. StOneman, K. ERICKSON et I. NeTtOn éd., The Alexander Romance..., p. 369-382.

48. A. FULINSKA, «Oriental Imagery and Alexander the Great in the Art of Central Asia », dans R.STONEMAn, K. ERICKSON et I. NetTon éd., The Alexander Romance..., p. 383-404.

49. http://www.library.rochester.edu/robbins/medieval-alexander. 
Histoires pour Roger, châtelain de Lille ; un troisième volume novateur intègre l'apport de la Renaissance et sa perception de la figure dans les lettres, l'histoire, les arts ; enfin, un volume thématique traite des voyages au paradis. Les quatre volumes suivants sont dédiés à la fascination pour Alexandre dans les littératures européennes entre le $\mathrm{X}^{\mathrm{e}}$ et le $\mathrm{XVI}^{\mathrm{e}}$ siècle.

L'Historiographie médiévale d'Alexandre le Grand ${ }^{50}$, ouvrage dirigé par Catherine Gaullier-Bougassas, aborde le problème de la figure d'Alexandre dans différents récits relatifs à sa légende qui proviennent d'adaptations d'œuvres anciennes ou de récits historiques dont certains textes ne sont pas encore édités.

Catherine Gaullier-Bougassas rappelle justement que la légende s'écrivit du vivant du personnage puis sous ses successeurs. La figure d'Alexandre a inspiré de nombreux travaux en Occident ayant valeur de mythe ; Claude Mossé ${ }^{51}$ et Pierre Briant ${ }^{52}$ en ont bien analysé les facettes, montrant les racines d'un personnage quasi universel en Europe et participant de l'imaginaire collectif, générant de nombreux travaux sur les origines d'un mythe à la fois lié à la personne du héros et aux nombreux historiens qui diffusèrent ce mythe. Dans cet océan où se mêlent et la légende et l'histoire, Catherine Gaullier-Bougassas évoque la façon dont les hommes de lettres du Moyen Âge pouvaient aborder le personnage et, surtout, les sources qui étaient à leur disposition en Europe de l'Ouest.

Alexandre étant à la fois un sujet historique et un sujet littéraire, sa légende est bâtie à partir de sources duelles, historiques et légendaires : Aristote, Xénophon puis ses compagnons, Callisthène, Ptolémée, Aristobule et les auteurs plus tardifs, Arrien ; mais aussi à partir de matériaux moins historiques et tout aussi fondamentaux, le plus important demeurant le Pseudo-Callisthène, qui confère à Alexandre les principaux stigmates d'une légende séculairement vivace, tant en Orient qu'en Occident.

En Occident, la tradition s'élabore à partir des textes de Diodore, Quinte-Curce et Justin, puis Paul Orose, mais aussi avec le Roman d'Alexandre du Pseudo-Callisthène ${ }^{53}$, sans compter la Bible, la Cité de Dieu, etc. Les auteurs médiévaux s'inspirent donc de sources variées qu'ils ne commencent à remettre en cause qu'à partir de la fin du XIV siècle avec l'émergence d'une critique historique. L'importance du passé sert à justifier

50. C. Gaullier-Bougassas éd., L'Historiographie médiévale d'Alexandre le Grand, Turnhout, 2011.

51. C. Mossé, Alexandre, la destinée d'un mythe, Paris, 2001.

52. P. BRIANT, Alexandre et l'héritage achéménide : quelques réflexions et perspectives, Paris, 2004 ; ID., De la Grèce à l'Orient, Alexandre le Grand, Paris, 1987 ; ID., Impérialismes antiques et idéologiecoloniale dans la France contemporaine : Alexandre le Grand modèle colonial, Paris, 1979 ; ID., Alexandre le Grand, Paris, 1974 ; etc.

53. G. BOunOuRE et B. SERRET trad., Le Roman d'Alexandre: la vie et les hauts faits d'Alexandre de Macédoine, Pseudo-Callisthène, Paris, 1992. 
le présent, car l'histoire est appréhendée par le rôle qu'elle peut jouer pour servir les intérêts chrétiens et le projet divin en général. Alexandre devient l'un des instruments de ce projet; son polymorphisme, ou tout au moins les aspects eschatologiques de la légende - Gog et Magog, quête du paradis - s'y prêtent parfaitement. Et sa figure christianisée et réappropriée devient celle d'un avatar du Dhü'l qarnayn oriental, préfigurant le Christ, comme chez Firdawsī et Nizāāī où il préfigure l'avènement de l'islam. Julius Valère semble réaliser cette fusion des deux traditions, comme le montre Jean-Pierre Callu alliant historia et mythistoria ${ }^{54}$, vérité et fiction, mais la version du Pseudo-Callisthène revit, elle, notamment sous la plume de Léon de Naples (Jean-Pierre Callu et Michel Festy ${ }^{55}$ ). Il est à noter que la figure d'Alexandre en Occident se teinte dès le $\mathrm{IV}^{\mathrm{e}}$ siècle d'une forte connotation religieuse, le héros devenant une figure de l'expansion du christianisme hors du limes et de la sauvegarde de la culture chrétienne. Ainsi, par exemple, le contexte des croisades et des luttes contre les musulmans influence grandement les auteurs espagnols, comme le restitue la compilation des Chroniques de Burgos analysées par Stéphanie Aubert ${ }^{56}$, où la christianisation du personnage, « agent de la volonté divine sur terre », prend tout son sens.

Les préoccupations d'une époque se retrouvent dans l'usage fait de la légende qui, associée aux sources historiques, donne naissance dans les différentes aires culturelles à une vision particulière du personnage, une acculturation en somme servant des desseins variés selon les auteurs, les destinataires ou le contexte lui-même, comme le montrent ici les œuvres dédiées à Alexandre (en France, en Angleterre, en Castille, dans les mondes germaniques). Héros civilisateur, combattant chrétien, roi philosophe, Alexandre devient, au cœur des littératures et dans les passages des œuvres qui s'attardent sur sa geste, une figure exemplaire, le prototype du miroir des princes qui apparaît en Orient au VIII ${ }^{\mathrm{e}}$ siècle et dont Mario Grinaschi avait bien percé l'identité de sujet privilégié ${ }^{57}$.

Les diverses contributions de ce volume questionnent ainsi, par aire linguistique, la naissance d'une historiographie, insistant chacune sur un auteur particulier ou une œuvre fondatrice de cette historiographie nationale d'Alexandre, issue de textes anciens comme dans la première traduction française de Valère Maxime (Graziella Pastore), sans oublier les influences

54. J.-P.CALlu, «Lire Julius Valère », dans C. Gaullier-Bougassas éd., L'Historiographie médiévale d'Alexandre le Grand..., p. 37-47; ID., Culture profane et critique des sources de l'Antiquité tardive, Rome, 2006 (CEF, 361), p. 565-582 ; et JULIUS VALÈrE, Roman d'Alexandre, trad. J.-P. CALLU, Turnhout, 2010.

55. "L'Alexandre latin », dans L'Historiographie..., p. 49-54.

56. «Alexandre le Grand dans Les Chroniques de Burgos », dans L'Historiographie..., p. $115-133$.

57. Voir n. 19. 
arabes sur l'historiographie occidentale (Faustina Doufikar-Aerts), de l'Historia de preliis en Italie (Michele Campopiano) ou de la Romance d'Alexandre en Arménie (Aram Topchyan). Un autre point de vue envisage la part du héros dans différentes œuvres, incluant parfois des extraits choisis : en Espagne, outre les Chroniques de Burgos (Stéphanie Aubert), puis le Libro de Alexandro en Castille au XV siècle (Rafael Beltrán), ou encore dans les compilations des Neuf preux (Anne Salamon). L'exemplarité de la figure est un trait important dans les histoires universelles françaises $\mathrm{du} \mathrm{XII}^{\mathrm{e}}$ au XV $\mathrm{XV}^{\mathrm{e}}$ siècle (Catherine Gaullier-Bougassas), dans les Revelationes du Pseudo-Méthode (Helena de Carlos), chez Vasque de Lucène (Hélène Bellon-Méguelle), ou encore chez deux historiens castillans, Fernán Pérez de Guzmán et Diego Rodríguez de Almela (Santiago Lopez-MartinezMoras) ; Monika Otter et Gerog Jostkleigrewe ont respectivement proposé une synthèse de la figure dans l'Angleterre et dans l'Allemagne médiévales.

Dans le sillage de cette réflexion sur l'introduction de la figure, des sources utilisées et des principales caractéristiques du mythe dans chacune des aires ou des œuvres fondatrices, Catherine Gaullier-Bougassas propose en guise de témoignage une édition commentée de L'histoire ancienne jusqu'à César ou Histoires pour Roger, châtelain de Lille, par Wauchier de Denain ${ }^{58}$. L'édition, réalisée à partir du ms. Paris, BnF, Français 20125, copié à Saint-Jean d'Acre vers 1280, réalisé pour les Lusignan du XIII ${ }^{\mathrm{e}}$ siècle, est augmentée d'un remaniement franco-italien du XIV ${ }^{\mathrm{e}}$ siècle qui permet de voir l'évolution de l'œuvre, et d'un texte d'Antoine Vérard datant de 1491, issu du «Volume d'Orose », comportant un récit sur Alexandre. L'auteur de l'Histoire ancienne, Wauchier de Denain, œuvre au début du XIII ${ }^{\mathrm{e}}$ siècle pour la cour de Flandre ; il est au service du châtelain de Lille, Roger IV, dans le contexte du conflit violent entre le roi de France Philippe Auguste et l'aristocratie de Flandres, après la mort du comte Baudouin IX, au sujet de la succession de ses filles, Jeanne et Marguerite de Flandre. De ce fait, le texte permet d'évaluer le poids de la contextualisation du roman, l'usage qui en est fait pour servir des desseins particuliers. Par ailleurs, on peut repérer les sources d'inspiration : Orose, Pierre le Mangeur, Justin, etc. Wauchier de Denain propose ici le premier récit en prose de la vie d'Alexandre qui revendique l'identité du héros dans son époque païenne. Catherine GaullierBougassas souligne le succès de l'œuvre chez les auteurs médiévaux.

Un autre volume est dédié à la réception de la figure à la Renaissance ${ }^{59}$, proposant ainsi une réflexion importante qui comble, comme le dit justement Corinne Jouanno, un vide historiographique. On y découvre les figures

58. L'Histoire ancienne jusqu'à César ou Histoires pour Roger, châtelain de Lille, de Wauchier de Denain. L'histoire de la Macédoine d'Alexandre le Grand, éd. C. GAULLIERBougassas, Turnhout, 2012.

59. C. Jounnno éd., Figures d'Alexandre à la Renaissance, Turnhout, 2012. 
d'Alexandre chez divers auteurs tels que Pétrarque (Rebecca Lenoir), Vasque de Lucène (Hélène Bellon-Méguelle), Pierre Amiot (Pascal Payen), de Rabelais à Étienne Pasquier (Emmanuelle Lacore-Martin), mais aussi la postérité de certains thèmes, dont celui de l'Ascension (Thibaut Maus de Rolley) ou des ancêtres d'Alexandre (Catherine Gaullier-Bougassas), et enfin les figures des femmes d'Alexandre à Fontainebleau sous François I ${ }^{\mathrm{er}}$ (Kathleen Wilson-Chevalier). On peut encore mesurer l'importance de la figure dans son réemploi en Scandinavie avec Olaus Magnus (Silvia Fabrizio-Costa), dans le traité de Loys Le Roy (Danièle Duport), et son impact dans diverses œuvres, dont l'emblématique (Anne Rolet), et dans l'œuvre de Montaigne (Nicolas Lombart).

\section{Les paradis d'Alexandre}

Outre les six volumes dédiés à l'historiographie, à l'analyse des œuvres et à l'édition de Wauchier de Denain, un huitième volume thématique est entièrement consacré cette fois aux Voyages d'Alexandre au paradis : Orient et Occident, regards croisés $^{60}$. Catherine Gaullier-Bougassas et Margaret Bridges évoquent les aventures paradisiaques du héros, les relations de ce dernier avec les espaces dévolus aux saints et aux prophètes, et les images du paradis qui se succèdent dans les différentes versions issues du Pseudo-Callisthène, mais aussi et surtout du Talmud de Babylone. C'est donc la conjonction de ces deux traditions que les contributeurs étudient à travers les aires et les œuvres médiévales dont chacun est le spécialiste, en évaluant la part de chacune d'entre elles, leur acculturation et le rôle de la fontaine de vie et des espaces paradisiaques dans les différentes traditions littéraires occidentales comme orientales médiévales. Les lecteurs mesureront l'impact du personnage sur les imaginaires médiévaux, les nombreux points de convergence dans les traditions littéraires, façonnant, du héros et de sa quête, une image somme toute pérenne malgré les quelques variations observées.

La première partie des contributions, intitulée «Les paradis d'Alexandre le Grand et l'horizon toujours renouvelé de la fiction », évoque l'origine de la légende d'Alexandre au paradis, essentiellement issue des textes rabbiniques et du Talmud, mais aussi l'importance du don d'un objet symbolique, la pierre, attestant l'impossibilité pour le héros de pénétrer l'espace sanctifié et de parvenir à son obsessionnel objectif, l’immortalité.

Catherine Gaullier-Bougassas, dans une contribution intitulée "Quête d'immortalité ou de salut des origines grecques et hébraïques aux réinterprétations orientales », présente minutieusement les différents

60. C. GAULlier-Bougassas et M. Bridges éd., Les Voyages d'Alexandre au paradis : Orient et Occident, regards croisés, Turnhout, 2013. 
éléments de cette enquête menée par les contributeurs en croisant les sources orientales et occidentales. La source de vie, l'intervention des prophètes Ilyās et Khāḍir/Khiḍr, la montagne paradisiaque, la pierre de paradis en sont les éléments majeurs. Si la quête de l'immortalité est bien présente dans le Roman du Pseudo-Callisthène lorsqu'Alexandre se déplace vers l'Occident du monde, dans le Talmud en revanche, le thème paradisiaque en dehors du pays des Bienheureux apparaît par l'évocation des abords du paradis lui-même. Les versions byzantines et serbes et les versions orientales, éthiopiennes et persanes citent, quant à elles, un avatar du jardin, la montagne paradisiaque, comme l'explicitera Marine Gaillard. Quant à la source de vie, l'ange ou la pierre, tous ces éléments sont bien présents, pour tout ou partie, dans les différentes traditions, germaniques, néerlandaises, anglaises, slaves.

Dans « La pierre du paradis et son enseignement dans les œuvres latines et romanes », Catherine Gaullier-Bougassas s'attache à présenter l'origine de cette tradition des portes du paradis, du voyage au paradis, un nouvel espace à conquérir pour le héros, à savoir l'Iter ad Paradisum, un texte inspiré du Talmud. Dans une perspective comparatiste, on peut rappeler qu'outre la montagne, le ciel est un autre espace associé au paradis que certains rois, Kay Kāwūs, le mythique roi de Perse, présent dans le Livre des rois de Firdawsī de Țūs, ou encore le roi Nemrod que les Qiṣaș alanbiy $\bar{a}$ ' ont immortalisé comme figure du tyran, ont, tout comme Alexandre dans la tradition occidentale, tenté de conquérir. Cette dimension, le ciel, a certes été étudiée par François de Polignac notamment ${ }^{61}$, mais elle peut rappeler l'aspect transgressif de la quête qui est irrémédiablement vouée à l'échec puisque le héros n'est ni saint, ni prophète, ce dont conviennent tous les contributeurs en se référant aux diverses traditions. D'ailleurs, les quêtes d'Alexandre - celles du paradis, sur terre, dans la mer, dans le ciel ne sont que l'occasion de l'informer, à l'exemple des monstra et autres merveilles, de la vanité de son désir de gloire, et des erreurs engendrées par un tempérament guidé par les passions et non par la foi. Comme le souligne Catherine Gaullier-Bougassas, lorsque le paradis n'est pas mentionné explicitement, il est évoqué par des symboles communs aux deux traditions, orientale et occidentale : arbres merveilleux, filles-fleurs, sources de vie, lieux paradisiaques, îles, etc. C'est cet espace paradisiaque qui est également analysé par Margaret Bridges dans les littératures germaniques, néerlandaises et anglaises.

La deuxième partie de l'ouvrage interroge les transmissions et les croisements culturels, au premier chef, le sens du voyage au paradis

61. F. DE Polignac, «Alexandre maître des seuils et des passages : de la légende antique au mythe arabe », dans L. HARF-LANCNER, C. KAPPLER et F. SUARD éd., Alexandre le Grand..., p. 215-225. 
et ses réécritures du roman originel issu du Talmud midrashim ancien et du Pseudo-Callisthène dans les traditions juives et orientales. Jean-Pierre Rothschild, dans «L'Iter ad Paradisum entre homélie rabbinique, roman, traité d'apologétique », présente Alexandre comme un être tyrannique, animé par le désir et la convoitise issus du regard, ' $\bar{a} y \bar{\imath} n$, mot qui signifie à la fois « œil » et « pierre », dont le poids se réduit une fois recouverte de poussière, révélant ainsi la vanité des biens de ce monde. D'ailleurs, Marco Di Branco, dans «Alessandro/Dū'l Qarnayn, il paradiso e la fonte della vita nella letteratura araba medievale », rappelle que les versions arabes ne comportent pas d'épisodes sur la vision du paradis car, comme on le sait, le seul personnage ayant eu accès au paradis est le prophète Muhammad lors de son Ascension céleste. En revanche, il faut mentionner la célèbre Sourate XVIII évoquant le fameux Dhū'l-Qarnayn (dont l'identité est discutée), qui associe le prophète Mūsā/Moïse à un homme bien curieux, Khị̣r, qu'Alexandre prend pour guide vers la source de vie dans les versions orientales. Sur ce point, on soulignera l'importance de la version syriaque dans les traditions arabes et l'ancienneté de la légende remontant à l'épopée de Gilgamesh, un personnage qui est ici totalement oublié. En outre, il faut rappeler le bel article de Françoise Aubaile-Sallenave sur ce même personnage ${ }^{62}$. René Bloch, dans «Alexandre le Grand et le judaïsme : la double stratégie d'auteurs juifs de l'Antiquité et du Moyen Âge », décrit comment le mythe du voyage d'Alexandre à Jérusalem a été inventé par des auteurs juifs afin de mettre en scène un topos, celui de l'empereur confronté au sage juif ; il précise aussi qu'il est difficile de prouver que l'Iter ad Paradisum est issu de la traduction d'un texte juif. Les sources orientales et leurs éléments particuliers, différents de ceux du Pseudo-Callisthène, se retrouvent enfin dans Alexandre de Paris, comme le montre Catherine Gaullier-Bougassas dans «Les eaux troublées de la quête d'Alexandre et les sources orientales du Roman d'Alexandre français », où sont présentés fontaine de vie, fleuve de mort et paradis terrestre.

La troisième partie évoque la quête sous le jour de l'élection ou de la révélation comme une initiation. Cet aspect avait été traité en 2001 dans le volume Alexandre le Grand, figure de l'incomplétude, édité par François de Polignac. Quelques compléments sont apportés par Patrick Gautier-Dalché, dans «Quatre notes sur Alexandre et la cartographie médiévale », qui évoque comment la cartographie médiévale installe des bornes tangibles de la quête, faisant de la mappemonde un espace-temps en lien avec le pouvoir, la souveraineté, la cosmologie, les territoires orientaux. Il analyse plus particulièrement celle d'Ebstorf, réalisée vers 1300 et hélas détruite en

62. F. Aubaile-SAlLENAVE, "Al-Khidr, "L'homme au manteau vert" en pays musulmans : ses fonctions, ses caractères, sa diffusion », dans Charmes et sortilèges. Magie et magiciens, Res orientales, 14 (2002), p. 11-36. 
1944. En outre, les peuples contenus au-delà de barrières, les inclusi, Gog et Magog, sont aussi signalés, comme d'ailleurs dans la cartographie arabe et persane. Il y aurait bon nombre de recoupements à signaler à ce propos ; notons, pour la cartographie orientale, la présence du mont Qāf, l'axe cosmique permettant l'accès au paradis, l'océan environnant, mais aussi et surtout la fameuse barrière d'airain que cosmographes et cartographes indiquent, telles les mappemondes d'Idrīsī, par exemple, et ce jusqu'au $\mathrm{XV}^{\mathrm{e}}$ siècle ; les monuments associés à Alexandre sont aussi représentés dans les cosmographies médiévales (celles de Dimashqī ou Gharnātīi, par exemple). La vocation sotériologique du héros est donc prégnante tant en Occident qu'en Orient médiéval, et on renverra sur cet aspect particulier l'ouvrage récent de Van Donzel et Schmidt cité ci-dessus.

D'autres traditions littéraires sont également éclairantes : l'analyse de Christophe Thierry sur «L'épisode du voyage au paradis dans le Strasburger Alexander (fin du XII ${ }^{\mathrm{e}}$-début du XIII ${ }^{\mathrm{e}}$ siècle) » insiste sur la dimension de circumnavigateur d'Alexandre, chère à Angelo Michele Piemontese pour ce qui concerne le roman persan ${ }^{63}$. En effet, au voyage terrestre, les épopées et romans de l'Iran médiéval ont ajouté un périple nautique qui mène Alexandre dans l'Océan indien, à Sarandib notamment, et jusqu'aux bornes du monde connu, dans le fameux océan environnant, ou bahr al-muhīt, que lui seul parvient à atteindre en passant par des détroits et des tourbillons périlleux. Les remarques sur le parcours initiatique, mais aussi sur les liens entre les versions allemandes et le contexte, l'empereur Frédéric II, autre figure négative semblable à l'antéchrist, sont fort intéressantes. Hélène Bellon-Méguelle détaille «Les imprévus du voyage d'Alexandre au paradis terrestre dans la littérature française de la fin du Moyen Âge ». On peut renvoyer ici aux travaux de Corin Braga sur le paradis perdu ${ }^{64}$. L'épisode chez les brahmanes, analysé par Elena Koroleva dans «Les brahmanes et le paradis dans les romans d'Alexandre russes », reproduit une variante christianisée, alors qu'à l'inverse le personnage d'Alexandre dans Le Rekontamiento del rey Ališandere en aljamiado serait, selon Émilie Picherot, bien conforme au roman du Pseudo-Callisthène.

Les thèmes étudiés dans la quatrième partie, « La démesure du désir et le désenchantement : un jugement ? », éclairent cependant les différences Orient-Occident, notamment sur la conception du paradis. Dans la tradition persane, analysée d'une part par Mario Casari, «Un lieu de traduction : Alexandre au paradis dans la tradition persane », et par Marina Gaillard

63. A.M. Piemontese, «Alexandre le circumnavigateur dans le roman persan de Tartusi », dans F. de Polignac éd., Alexandre le Grand, figure de l'incomplétude, Mélanges de l'École Française d'Athènes et de Rome, 112 (2001/1), p. 97-112.

64. C. Braga, Le Paradis interdit au Moyen Âge. La quête manquée de l'Éden oriental, Paris, 2004 ; ID., La Quête manquée de l'Avalone occidentale. Le Paradis interdit au Moyen Âge, vol. 2, Paris, 2006. 
d'autre part, « D'un bout du monde à l'autre : lieux paradisiaques et terres ultimes dans le roman d'Alexandre en prose de l'Iran classique », le paradis du monde musulman, conformément à son orthodoxie, n'est pas sur terre. On ne peut donc s'y rendre ; toutefois, les lieux paradisiaques existent mais ils présentent tous une certaine ambiguïté ; en outre, monstres et merveilles sont, eux, envoyés par les cieux pour instruire le héros (on relira à ce sujet l'article de Claire Kappler ${ }^{65}$ ). Mario Casari développe un aspect important ayant trait au polyglottisme d'Alexandre et à ces fameux traducteurs qui habiteraient la cité des bienheureux de Niẓāmī, et pour lesquels il propose une analogie pertinente entre traducteurs et brahmanes. Alexandre est aussi un personnage que l'on peut contextualiser ou se réapproprier dans les lettres allemandes, écossaises ou anglaises pour les besoins d'une cause littéraire ou politique spécifique, comme l'explicite Jean-Claude Mühlethaler dans «Échec amoureux et échec politique : le remploi du Voyage au paradis dans le Chevalier errant de Thomas III de Saluces) », Anna Caughey et Emily Wingfield dans «Conquest and Imperialism, Medieval Scottish Context of Alexander Journey to Paradise », ou encore Margaret Bridges dans « Five Late Middle English Versions of the Narreme of Alexander's Wondrous Gift». Dans les œuvres nationales analysées par les contributeurs, ces passages renvoyant à l'Iter ad Paradisum servent véritablement, comme le dit Margaret Bridges, d'exempla, des temps forts de la littérature morale ou chrétienne médiévale.

L'érudition des contributions, les bibliographies presque exhaustives témoignent du renouvellement de la recherche depuis les années 2000 et font de cet ouvrage une contribution importante à l'histoire d'Alexandre et de son imaginaire oriental comme occidental. Pourtant, on regrettera que l'image même d'Alexandre, son étude iconographique, ne fasse pas l'objet du même intérêt. Or, quoi de plus évocateur visuellement que l'image du paradis ?

\section{La fascination pour Alexandre le Grand}

Une seconde série d'ouvrages dans la collection - quatre volumes concerne cette fois la réception d'Alexandre en Europe. Les tomes 1 et 4 sont deux volumes de référence, fort utiles pour le chercheur travaillant sur les corpus européens d'Alexandre. Le tome $1^{66}$ présente en deux parties le panorama des littératures européennes transmettant les romans d'Alexandre dans la littérature latine en langue française, italienne, anglaise, allemande, néerlandaise, scandinave, tchèque, russe et serbe, arménienne, gréco-

65. C. KAPPLER, «Alexandre et les merveilles...».

66. C. Gaullier-Bougassas éd., La Fascination pour Alexandre le Grand dans les littératures européennes ( $X^{e}$-XVI $e^{e}$ siècle). Réinventions d'un mythe, t. I, Turnhout, 2014. 
byzantine par le prisme des sources du Pseudo-Callisthène, Quinte-Curce, les histoires universelles, la littérature didactique et morale, du Secretum secretorum et les écritures humanistes. Le tome $4{ }^{67}$ traite des réécritures médio-latines et vernaculaires : Léon de Naples et l'Historia de preliis, Gauthier de Châtillon, le Roman d'Alexandre composé en français, puis en italien, en aljamiado, en hébreu, dans les lettres anglaises et allemandes, néerlandaises, scandinaves, tchèques, russes, arméniennes et grecques tardives. Ce panorama impressionnant se double dans le tome 4 d'un répertoire non moins impressionnant des manuscrits cités dans les contributions avec indications sur les auteurs, copistes, contenu de l'œuvre et bibliographie spécifique.

Deux volumes méritent pour l'historien médiéviste une attention particulière : il s'agit du tome 2 sur le pouvoir royal d'Alexandre et du tome 3 sur l'imaginaire de la connaissance savante et des lointains exotiques.

Le tome 2, consacré à la réinvention du mythe, questionne l'impact de la figure d'Alexandre comme modèle dans les idéologies de la royauté et la littérature des miroirs des princes ${ }^{68}$. Le discours sur Alexandre est ainsi instrumentalisé pour servir des objectifs politiques parfois christianisés, comme celui de Gauthier de Châtillon dans l'Alexandreis. Ces romans posent le problème des destinataires (haut clergé, aristocratie, roi), qui influent sur l'orientation du texte et ses objectifs. L'auteur y déploie ses talents comme conseiller moralisateur, auteur d'un miroir du prince, serviteur de la propagande officielle, voire en homme de lettres innovant. Le roman permet ainsi, par ses ambitions, de montrer les limites du pouvoir et ses aléas, de faire émerger une nouvelle figure, le sage conseiller, auteur que l'Orient connaît parfaitement depuis l'époque omeyyade et le Secretum secretorum et qui s'affirme à l'époque seldjoukide avec des vizirs hommes de lettres, tels Nizāam al-Mulk à la fin du XI ${ }^{\mathrm{e}}$ siècle à Ispahan ou Firdawsī de Ṭūs à Ghazna au début du XI ${ }^{\mathrm{e}}$ siècle ${ }^{69}$. L'image d'Alexandre se construit autour de plusieurs paradigmes : la connaissance et le savoir, les liens avec le divin et les vertus royales, celles que l'on retrouve par exemple chez Nizāmī de Ganja dans l'Iskandar Nāma au XII ${ }^{\mathrm{e}}$ siècle, et qui n'ont pas varié en Occident.

La royauté d'Alexandre dans la littérature médio-latine est présentée par Alexandru Cizek et Jean-Yves Tilliette. Léon de Naples rapporta

67. C. Gaullier-Bougassas éd., La Fascination pour Alexandre le Grand dans les littératures européennes..., t. IV : Répertoire du corpus européen, Turnhout, 2014.

68. C. GaUllier-Bougassas éd., La Fascination pour Alexandre le Grand dans les littératures européennes..., t. II, $3^{\mathrm{e}}$ partie: Le pouvoir royal d'Alexandre : littérature et politique. Les auteurs, leurs mécènes et leurs publics, Turnhout, 2014.

69. On reverra d'ailleurs l'admirable ouvrage de Charles-Henri de Fouchécour qui a bien montré l'impact de la figure d'Alexandre sur la littérature de miroirs au prince jusqu'au $\mathrm{XV}^{\mathrm{e}}$ siècle : voir supra, n. 25. 
de Constantinople, au $\mathrm{X}^{\mathrm{e}}$ siècle, un manuscrit du Roman d'Alexandre du Pseudo-Callisthène et en composa une version, l'Historia de preliis, base de nombreuses compositions romanesques. Les deux contributeurs analysent au préalable la façon dont, sous l'influence de l'Église, l'idéal de la royauté s'est modifié, affectant la littérature des miroirs des princes de Jonas d'Orléans à Jean de Salisbury et Giraud de Barri, qui chacun émettent une vision différenciée d'Alexandre, parfois même négative. Dans l'Historia de preliis et ses différentes versions, on retrouve le portrait dressé par le Pseudo-Callisthène plus que par Quinte-Curce, car parmi les qualités du souverain idéal sont mis en exergue le courage, mais aussi la sapientia acquise auprès des sages et d'Aristote. Le roi sage et philosophe ne dédaigne pas de converser avec les brahmanes ; conciliateur et médiateur, astucieux au combat, il peut aussi être déloyal et parfois rusé. Les symboles du pouvoir, trône de joyaux à sept degrés, et le symbolisme des chiffres en général renvoient à l'image du cosmocrator. En comparaison, l'Alexandreis de Gauthier de Châtillon relève du miroir des princes (destiné peut-être à Philippe Auguste), mettant en valeur l'enseignement d'Aristote et faisant du héros un preux chevalier qui doit vaincre et dépasser ses travers.

Selon Catherine Gaullier-Bougassas et Hélène Bellon-Méguelle, l'Alexandre français est d'abord, depuis le XII siècle, un modèle idéal qui s'impose par ses qualités personnelles; au XIII ${ }^{\mathrm{e}}$ et au XIV ${ }^{\mathrm{e}}$ siècle, c'est davantage le souvenir de cette figure qui subsiste au travers des genres littéraires ou d'adaptations d'écrits antérieurs (comme ceux de Jean de Salisbury notamment). Mais, quelle que soit la période, le pouvoir royal est au centre des préoccupations des auteurs. Chez Alexandre de Paris, le roi est lettré, instruit en rhétorique, dialectique et quadrivium, inventif et généreux. La question de sa naissance n'est pas décisive mais, en revanche, la domestication de ses passions et de ses désirs impose le respect.

Perceforest présente une figure réinventée d'Alexandre comme ancêtre tutélaire de la monarchie anglaise, et l'on voit l'intérêt de cette dernière pour cette figure à travers le goût de Philippa de Hainaut pour les manuscrits, et d'Édouard III pour Alexandre auquel il s'identifie. On voit par ailleurs que d'autres romans, tels ceux de Jean Wauquelin au XV $\mathrm{V}^{\mathrm{e}}$ siècle ou de Thomas de Kent, donnent une image différente, celle d'un roi pieux au service de Dieu. On trouvera ainsi les analyses de nombreux romans, celles de Wauchier de Denain, de Baudoin d'Avesnes, de Jean de Vignay, de Jean de Courcy, de Jean Mansel, mais aussi les traductions de la cour de Bourgogne.

En Italie, d'après l'analyse des œuvres médiévales et de la Renaissance effectuée par Michele Campopiano, la figure est indéniablement marquée par l'influence de Dante, Pétrarque et Boccace. Toutefois, les nombreux récits qui lui sont dédiés présentent un portrait original, adapté aux problématiques italiennes, notamment à travers la promotion d'une royauté 
impériale à portée universelle. La figure chevaleresque, elle, est présente dans les textes de vulgarisation issus de l'Historia de preliis; cette figure est également repensée à la Renaissance, chez Machiavel notamment.

À la cour de Castille et du León, aux XIII ${ }^{e}$ et XIVe siècles, d'après Amaia Arizaleta, ce sont les clercs qui, composant des miroirs des princes, s'inspiraient de textes écrits antérieurement (au XIII ${ }^{\mathrm{e}}$ siècle le plus souvent), tel le Libro de Alexandre, une œuvre qui assure la promotion d'une figure chrétienne, conquérante, juste, mais où se pose le problème de la filiation, évoquant les royales figures de Ferdinand III et surtout d'Alphonse VIII, pour lequel l'œuvre fut écrite. Mais c'est Alphonse X, son petit-fils, qui en assure la promotion comme roi savant et courageux dont le portrait se retrouve fondu à la figure d'Alexandre dans les Bocados de oro de Mubaššir ibn Fatik et comme figure remarquable de son modèle politique de roi savant, guerrier, intéressé par les sciences occultes que l'on retrouve en Castille dans les Bocados de oro et dans le Poridad de las poridades, où la connaissance des sciences occultes est marquée. Sous Alphonse XI (m. 1350), un règne moins connu qui a néanmoins suscité une littérature consacrée à Alexandre, l'auteur du Poema de Alfonso XI (1348), Ruy Yánez, marque le rapport à Dieu, la dimension spirituelle, faveur divine. Émilie Picherot compare le Rekontamiento del rey Ališandera, appartenant à la littérature morisque (et dont la source est la Qissat Dhulqarnayn du IX ${ }^{\mathrm{e}}$ siècle), au récit de Țabarī, et expose le modèle idéal de souverain que les Morisques ont élaboré en lui donnant une identité orientale marquée.

Margaret Bridges analyse, quant à elle, le Buik of King Alexander the Conquerour de Gilbert Hay, c'est-à-dire «la somme des traditions romanesques»: elle la présente comme un modèle qui n'est pas exclusivement aristocratique mais destiné à tous les hommes. Dans le Saint-Empire, de nombreux récits sont analysés par Marie-Sophie Masse et Christophe Thierry, dont le Vorauer Alexander, datant du dernier quart $\mathrm{du} \mathrm{XII}^{\mathrm{e}}$ siècle, et dans sa continuation, le Strasburger Alexander, on dresse le portrait d'une figure idéale et atemporelle de la royauté, joignant valeurs aristocratiques et cléricales. Au XIII ${ }^{\mathrm{e}}$ siècle, l'Alexander de Rudoph présente Alexandre comme un être sage, parfois cruel, mais d'une piété exemplaire, même s'il est difficile d'y voir une association avec les Hohenstaufen. Seifrit, dans son Alexander, glorifie, lui, la figure de l'empereur élu, forme de soutien à l'Empire universel servant la théorie de la translatio imperii, une vision que l'on retrouve dans le Grosser Alexander au XIV siècle.

Éloïse Adde-Vomáčka démontre comment, en Bohème, Alexandre est devenu une figure de la propagande royale aux XIII e et XIV ${ }^{\mathrm{e}}$ siècles notamment, pour la famille des Luxembourg. Charles IV usa de la figure comme modèle de gouvernement, et les fresques du château de Prague exposaient la figure d'Alexandre, sans compter le camée de la croix du couronnement en 1357, ou encore la création d'un faux privilège du scribe 
Jan... Parmi les différentes œuvres dédiées à Alexandre, l'Alexandreida, adaptation de l'œuvre de Gautier de Châtillon, diffuse une idéologie nobiliaire qui invite à lire l'œuvre comme un intertexte exposant les crises politiques de l'époque. Mais il faut signaler que la figure d'Alexandre inspira également la noblesse qui, parfois, y trouva de quoi combattre l'absolutisme monarchique. Aux XIV et $\mathrm{XV}^{\mathrm{e}}$ siècles, Alexandre servit d'autres desseins, notamment ceux du mouvement hussite et du Renouveau national et panslaviste.

Elena Koroleva évoque le cas du roman en Russie, où Alexandre est une figure positive, qui sert à valoriser celle d'Alexandre Nevski ; Alexandre est également considéré comme un roi modèle et glorieux, mais dont les limites sont celles des dérives de l'autocratie (avec Alexandre de Serbie, par exemple). En Arménie, la postérité du roman, présentée par Giusto Traina, réside dans l'Histoire d'Arménie de Moïse de Khorène. En Grèce, les trois adaptations analysées par Catherine Jouanno sont tardives (XVI $I^{\mathrm{e}}$ siècle) et, dans le contexte de l'Empire ottoman assimilé aux Perses, Alexandre devient un héros remarquable, très chrétien ou marqué par les valeurs chrétiennes, notamment dans les passages de La Rimada dédiés aux méditations sur la vanité des ambitions et sur la mort.

Le tome 3 de la même série, dédié à L'Alexandre scientifique et aventurier $^{70}$, expose comment la connaissance du monde passe par la découverte des mirabilia, mais aussi par celle des choses secrètes indiquées notamment par Aristote dans le Sirr al-asrār ou Secretum secretorum. Les contributions explorent principalement les différentes conceptions de la merveille selon les traditions. Il est dommage que l'imaginaire des savoirs occultes, qui est bien connu pour la France, n'ait pas été développé pour les autres aires européennes (excepté l'Italie).

La dernière partie, consacrée aux raisons du succès, est fondamentale, car on s'interroge sur l'abondance des réécritures, des manuscrits et de l'engouement européen pour ce personnage mythique. Tous les contributeurs soulignent le thème de la royauté, des savoirs, mais aussi la persistance de l'ambiguïté d'une figure tutélaire qui sert à la fois des objectifs politiques (celui des miroirs des princes) tout en suscitant des réserves, notamment dans le champ de la morale et de l'éthique chrétienne. Et n'est-ce pas justement parce qu'elle se trouve entre histoire et fiction que la figure d'Alexandre provoque ce malaise, la volonté de faire cadrer le personnage romanesque avec un personnage historique qui en est, au fond, très éloigné ?

70. C. Gaullier-Bougassas éd., La Fascination pour Alexandre le Grand dans les littératures européennes..., t. III, $4^{\mathrm{e}}$ partie : L'Alexandre scientifique et aventurier. Un imaginaire de la connaissance savante et des lointains exotiques, Turnhout, 2014. 
Depuis une dizaine d'années, les travaux sur le Roman d'Alexandre ont montré la nécessité de se pencher sur la transmission et la réception des textes littéraires issus de l'Antiquité, tant dans le monde oriental qu'occidental. La légende d'Alexandre constitue un cas particulier car elle fédère à la fois le domaine politique, la connaissance du monde, les sciences occultes et enfin la littérature. Par la fascination que le personnage continue d'exercer, le Roman d'Alexandre possède encore de beaux jours devant lui. Pourtant, s'il constitue aujourd'hui un thème de recherche interdisciplinaire pour les humanités, la partie visuelle notamment reste encore à explorer. En effet, depuis l'étude fondamentale de David J. A. Ross ${ }^{71}$, le thème a connu peu d'engouement, en dehors de la récente thèse de Maud Perez-Simon ${ }^{72}$. Toutefois, la richesse et les nombreuses pistes de réflexion offertes par les corpus présentés dans Alexander Redidivus permettraient d'aborder ces mêmes corpus dans leurs illustrations cette fois, sous forme analytique ou thématique, à l'image du vol ${ }^{73}$. Signalons, en outre, que d'autres supports, à l'instar des textiles ${ }^{74}$, conjugués aux textes historiques et littéraires, offrent de précieuses sources pour l'histoire des imaginaires culturels et politiques $^{75}$.

Anna Caiozzo - Université Paris-Diderot

71. D. J. A. Ross, Alexander Historiatus : a Guide to Medieval Illustrated Alexander Literature, Londres, 1963.

72. M. PEREZ-SiMON, Mise en roman et mise en image : les manuscrits du «Roman d'Alexandre » en prose, Paris, 2015.

73. V. M. SCHMIDT, A Legend and its Image : the Aerial Flight of Alexander the Great in Medieval Art, Groningue, 1995.

74. F. Barbe, L. Stagno, E. Villari éd., L'Histoire d'Alexandre le Grand dans les tapisseries au XVe siècle: fortune iconographique dans les tapisseries et les manuscrits conservés : la tenture d'Alexandre de la collection Doria Pamphilj à Gênes, Turnhout, 2014.

75. K. Dimitrova, M. L. Goehring, Dressing the Part: Textiles as Propaganda in the Middle Ages, Turnhout, 2014. 